\title{
Enantioselective crystallization of diglycerol dicarbonate: impact of the microstructure on polyhydroxyurethane properties
}

Fiona Magliozzi ${ }^{1}$, Arthur Scali ${ }^{1}$, Guillaume Chollet ${ }^{2}$,Etienne Grau ${ }^{1 *}$, Henri Cramail $^{{ }^{*}}$

${ }^{1}$ Univ. Bordeaux, CNRS, Bordeaux INP, LCPO, UMR 5629, F-33600, Pessac, France, cramail@enscbp.fr

${ }^{2}$ ITERG, 11 rue Gaspard Monge, 33610 Canéjan, France

Keywords: Diglycerol Dicarbonate, stereoisomers, crystallization, polyhydroxyurethanes

\begin{abstract}
Bicyclic carbonates are precursors employed in non-isocyanate polyurethane syntheses, namely polyhydroxyurethanes (PHUs) and, among them, ether-activated biobased diglycerol dicarbonate (DGDC) is widely investigated. Herein, we report the original separation of DGDC stereoisomers thanks to a recrystallization procedure, leading to enantiomers having different crystal lattices, i.e. square transparent crystals and needle like structures, respectively. In this study, the separation and the characterization of the two crystal structures is first discussed and, secondly, the impact of the stereochemistry of DGDC on the corresponding PHUs properties investigated.
\end{abstract}

\section{INTRODUCTION}

DGDC is a biobased ether-activated 5 membered-bisCyclicCarbonate (bisCC), commonly obtained in one step from diglycerol and dimethylcarbonate. ${ }^{1}$ However, the first reported synthesis of DGDC was based on the carbonation of diglycidyl ether of diglycerol ; the authors obtaining the DGDC as a crystalline powder characterized by a melting temperature of 62 $63{ }^{\circ} \mathrm{C} .^{2}$

In 2015, Tryznowski et al. and Van Velthoven et al., separately, described the synthesis of DGDC in one step from diglycerol and its use in the course of polyhydroxyurethanes (PHUs) synthesis. ${ }^{3,4}$ After a recrystallization in ethyl acetate, Tryznowski et al. obtained DGDC as a 
powder with a melting temperature of $65.9^{\circ} \mathrm{C}$. The work up developed by Van Velthoven et al. used a washing step with water leading to the formation of DGDC powder with a melting temperature of $64.8^{\circ} \mathrm{C}$.

Bossion et al. reported in 2018 DGDC-based PHUs, synthesized with assistance of different catalysts. The bisCC monomer was obtained from diglycerol and purified by recrystallization in cold methanol. ${ }^{5}$ Following this DGDC synthetic procedure, our group described the benefit brought by the reactive extrusion process, on DGDC-based PHUs synthesis. ${ }^{6}$

Very recently, Schimpf et al. reported DGDC synthesis based on the carbonation of diglycidyl ether of diglycerol and operated with a recrystallization of DGDC in a mixture of acetone and diethyl ether for $48 \mathrm{~h}$ at $-20{ }^{\circ} \mathrm{C} .{ }^{1}$ Interestingly, DGDC was recovered as a white powder with a melting temperature of $74^{\circ} \mathrm{C}$. The authors claimed the highest melting temperature never recorded in literature for such bisCC. Experimental conditions dealing with DGDC syntheses and purification procedures together with the corresponding physico-chemical properties are available in SI Table SI.1.

Based on these literature data, we investigated further the recrystallization and purification of DGDC. Indeed, we report in this work, the separation and characterization of DGDC stereoisomers. The study demonstrates that $\mathrm{R}, \mathrm{S} / \mathrm{S}, \mathrm{R}$ and $\mathrm{R}, \mathrm{R} / \mathrm{S}, \mathrm{S}$ enantiomers crystallize according to two different crystal systems, appearing as needle like crystals (DGDC-NeC) and square like crystals (DGDC SqC), respectively. Finally, the separated stereoisomers were tested in the course of aminolysis with 1,10-diaminodecane, hexamethylenediamine, and m-xylylene diamine and then used as precursors of polyhydroxyurethanes. The impact of DGDC microstructure on the PHU properties was evaluated.

\section{EXPERIMENTAL SECTION}

\section{Materials.}


The reactants such as diaminodecane (10DA), xylylenediamine (xy-DA), and diglycerol were purchased from TCI, Europe. Sodium methoxide (NaMeO) was provided by Alfa Aesar. Dimethylcarbonate (DMC, extra dry, 99\%), and hexamethylenediamine (6DA), were obtained from Accros. Solvents like dimethylformamide (DMF) (for chromatography), methanol, and diethyl ether were ordered from VWR. Deuterated dimethyl sulfoxide (DMSO) was purchased from Eurisotop.

\section{Instruments \& Methods.}

Nuclear Magnetic Resonance. ${ }^{1} \mathrm{H}$ and ${ }^{13} \mathrm{C}$ nuclear magnetic resonance (NMR) spectra were recorded on a Bruker ADVANCE 400 spectrometer $\left(400.20\right.$ and $100.63 \mathrm{MHz}$ for ${ }^{1} \mathrm{H}$ and ${ }^{13} \mathrm{C}$, respectively) using DMSO-d6 as a solvent, at room temperature. The polymerization extent was determined by monitoring the carbonate functions conversion according to NMR spectra, following the disappearance of signals belonging to the carbonate. The peak corresponding to the protons in $\alpha$ position of the urethane bond was used as the internal reference. No internal or external standard was used for the NMR analysis.

Size Exclusion Chromatography. Polymer molar masses were determined by size exclusion chromatography (SEC) using DMF (DMF + lithium bromide $\operatorname{LiBr} 1 \mathrm{~g} / \mathrm{L}$ ) as the eluent. Measurements in DMF were performed on an UltiMate 3000 system from Thermoscientific equipped with diode array detector DAD. The system also includes a multiangle light scattering detector MALLS and differential refractive index detector dRI from Wyatt Technology. Polymers were separated on a system composed of two GF310 + 510 Asahipak columns containing a poly(vinyl alcohol) phase $(7.5 \times 300$, exclusion limit: 300 000). Columns temperature was held at $50{ }^{\circ} \mathrm{C}$. Polystyrene was used as the standard and the calibration method is conventional.

Thermogravimetric Analyses (TGAs). TGAs were performed on the TGA-Q500 system from TA Instruments at a heating rate of $10{ }^{\circ} \mathrm{C} \min ^{-1}$ under a nitrogen atmosphere from room 
temperature to $600{ }^{\circ} \mathrm{C}$ and then under air atmosphere from 600 to $700{ }^{\circ} \mathrm{C}$. Differential Scanning Calorimetry.

Differential scanning calorimetry (DSC). For PHU $\mathrm{T}_{\mathrm{g}}$ determination, thermograms were measured using a DSC Q100 apparatus from TA Instruments. For each sample, two heating cycles were performed from -40 to $180{ }^{\circ} \mathrm{C}$ at $10{ }^{\circ} \mathrm{C} \mathrm{min}$. The glass transition and melting temperatures of crystalline domains were calculated from the second heating run. DGDC melting temperatures were determined according to DSC first heating cycle $\left(-40^{\circ} \mathrm{C}\right.$ to $\left.150{ }^{\circ} \mathrm{C}\right)$, at a rate of $10{ }^{\circ} \mathrm{C} / \mathrm{min}$. Phase diagram was drawn according to melting temperature obtained with DSC first heating cycle at $5{ }^{\circ} \mathrm{C} / \mathrm{min}$.

DGDC Synthesis. DGDC was synthesized according to the previously reported synthesis. ${ }^{6}$ DGDC was prepared from diglycerol with a $40 \%$ yield through a one-step procedure. In a round-bottom flask, $76 \mathrm{~g}$ (1 eq.) of diglycerol were introduced and dried under vacuum during $2 \mathrm{~h}$ at $90{ }^{\circ} \mathrm{C}$. Then, nitrogen was flushed into the vessel and $1.24 \mathrm{~g}$ ( 0.05 eq.) of sodium methoxide catalyst was added under stirring. A condenser was set up above the flask and 5 eq. $(231 \mathrm{~mL})$ of the second reactant, DMC, was added. The DMC also plays the role of the reaction solvent. The temperature was then set at $110^{\circ} \mathrm{C}$. After $48 \mathrm{~h}$ of reaction, the mixture was filtered to remove the sodium methoxide and concentrated by evaporation.

Procedure 1. Two successive recrystallizations in cold methanol $\left(-80{ }^{\circ} \mathrm{C}\right)$. DGDC was recovered by filtration on paper filters and dried under vacuum at room temperature.

Procedure 2. Temperature based recrystallization. A $55 \% / 45 \%$ mixture of acetone/diethyl ether was added to the reaction crude. After $48 \mathrm{~h}$ at $-20{ }^{\circ} \mathrm{C}$, Square like crystals (DGDC-SqC) and Needle like crystals (DGDC-NeC) were recovered.

Procedure 3. Temperature based separated recrystallizations. After concentration, the reaction crude is placed at $18{ }^{\circ} \mathrm{C}$ for 3 days. DGDC-SqC are formed in the bottom of the flask and can 
be recovered and dried. Afterward, $500 \mathrm{ml}$ of acetone and $450 \mathrm{ml}$ of diethyl ether are added to residual reaction crude (after DGDC-SqC removal) and the flask is placed at $-20{ }^{\circ} \mathrm{C}$. After $48 \mathrm{~h}$ needles appeared and can be recovered. This protocol allows the proper separation and recovery of the two different DGDC crystal shapes, DGDC-SqC first and then DGDC-NeC.

${ }^{1} \mathrm{H}$ NMR (DMSO-d6, $25{ }^{\circ} \mathrm{C}, 400 \mathrm{MHz}$, carbon numbers are displayed in Figure 1): DGDC-Pow, $\delta(\mathrm{ppm}): 4.93\left(\mathrm{~m}, 2 \mathrm{H}, \mathrm{H}_{2}\right), 4.52,\left(\mathrm{t}, 2 \mathrm{H}, \mathrm{H}_{1}{ }^{\text {cis or trans }}\right), 4.25$ (ddd, $\left.2 \mathrm{H}, \mathrm{H}_{1}{ }^{\text {cis or trans }}\right), 3.65-3.79$ (m, 4H, $\left.\mathrm{H}_{3}\right)$. DGDC-SqC, $\delta(\mathrm{ppm}): 4.93\left(\mathrm{~m}, 2 \mathrm{H}, \mathrm{H}_{2}\right), 4.52\left(\mathrm{t}, 2 \mathrm{H}, \mathrm{H}_{1}{ }^{\mathrm{cis}}\right), 4.23$ (dd, 2H, $\left.\mathrm{H}_{1}{ }^{\text {trans }}\right), 3.65-$ 3.79 (ddd, 4H, $\mathrm{H}_{3}$ ). DGDC-NeC, $\delta(\mathrm{ppm}): 4.93\left(\mathrm{~m}, 2 \mathrm{H}, \mathrm{H}_{2}\right), 4.52\left(\mathrm{t}, 2 \mathrm{H}, \mathrm{H}_{1}{ }^{\mathrm{cis}}\right), 4.26(\mathrm{dd}, 2 \mathrm{H}$, $\left.\mathrm{H}_{1}{ }^{\text {trans }}\right), 3.66-3.77\left(\mathrm{ddd}, 4 \mathrm{H}, \mathrm{H}_{3}\right)$

${ }^{13} \mathrm{C}$ NMR (DMSO-d6, $\left.25{ }^{\circ} \mathrm{C}, 100 \mathrm{MHz}\right):$ DGDC-Pow, $\delta$ (ppm): 155.35 - 155.30 (C4), 75.82 75.28 (C2), 70.830 - 70.857 (C3), 66.35 (C1). DGDC-SqC, $\delta$ (ppm): 155.35 (C4), 75.82 (C2), 70.830 (C3), 66.35 (C1). DGDC-NeC, $\delta$ (ppm): 155.30 (C4), 75.28 (C2), 70.857 (C3), 66.35 $(\mathrm{C} 1)$.

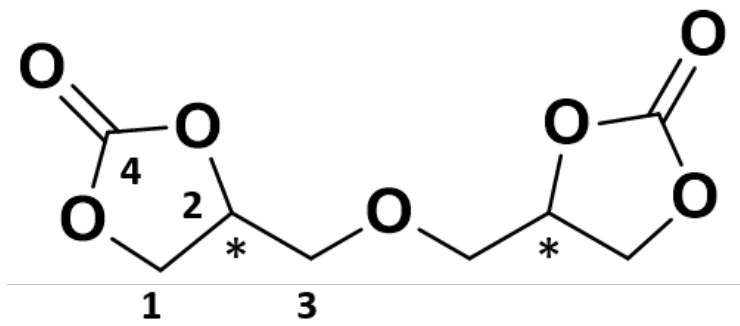

Figure 1. DGDC structure and carbon numbers for ${ }^{13} \mathrm{C}$ attribution. Asterisk represent asymmetric carbons.

Kinetic study on model reactions. 74.4mg (1 eq.) of DGDC-SqC and DGDC-NeC are dissolved in $1 \mathrm{ml}$ of DMSO-d6. Hexylamine $(69.07 \mathrm{mg}, 2$ eq.) is added and the system is quickly mixed before $500 \mu \mathrm{L}$ are taken and injected into an NMR tube. Monitoring of the aminolysis reaction was performed by ${ }^{1} \mathrm{H}$ NMR at $50{ }^{\circ} \mathrm{C}$ during $20 \mathrm{~h} .{ }^{1} \mathrm{H}$ NMR analyses were performed every 10 minutes during the first hour of reaction and then every hour until $20 \mathrm{~h}$. The reaction extent is calculated according to the disappearance of protons related to cyclic carbonate moieties and 
kinetic rate constants are determined thanks to $1 / C-1 / C_{0}$ curves, drawn from the initiation and up to 55 minutes of reaction.

Standard Procedure for Polymerization with Mechanical Stirring. Bis-CCs (1 eq., 0.5 g) were introduced in Schlenk tubes and heated at the desired temperature (oil bath). Then, the paddle stirrer for mechanical stirring was added to the Schlenk tube. The steel mixing paddle is screwed to a IKA RW20 Digital instrument. The bis-CCs were melted and stirred during $5 \mathrm{~min}$, and the appropriate amount of diamine (1 eq.) was added under stirring. This method allows the immediate mixing of the diamine with the melted bis-CC, preventing its sublimation. After the appropriate reaction time, the stirring was stopped, the Schlenk tube cooled down to room temperature and the samples were analyzed.

Electrospray (ESI) - Qexactive. Mass spectra were performed by the CESAMO (Bordeaux, France) on a Qexactive mass spectrometer (Thermo). The instrument is equipped with an ESI source and spectra were recorded in the negative/positive mode. The spray voltage was maintained at $3200 \mathrm{~V}$ and capillary temperature set at $320^{\circ} \mathrm{C}$. Samples were introduced by injection through a $20 \mu \mathrm{L}$ sample loop into a $300 \mu \mathrm{L} / \mathrm{min}$ flow of methanol from the LC pump. MALDI analysis. MALDI-MS spectra were performed by the CESAMO (Bordeaux, France) on an Autoflex maX TOF mass spectrometer (Bruker Daltonics, Bremen, Germany) equipped with a frequency tripled Nd:YAG laser emitting at $355 \mathrm{~nm}$. Spectra were recorded in the positiveion mode using the reflectron and with an accelerating voltage of $19 \mathrm{kV}$. Samples were dissolved in THF at $10 \mathrm{mg} / \mathrm{ml}$. The IAA matrix (Indole acrylic acid) solution was prepared by dissolving $10 \mathrm{mg}$ in $1 \mathrm{ml}$ of THF. A MeOH solution of cationisation agent (NaI, $10 \mathrm{mg} / \mathrm{ml}$ ) was also prepared. The solutions were combined in a 10:1:1 volume ratio of matrix to sample to cationisation agent. One to two microliters of the obtained solution was deposited onto the sample target and vacuum-dried. 
X-Ray diffraction The data for the crystal structure of DGDC was collected at the IECB on a Bruker microstar X8 PROTEUM with a classical kappa geometry and Platinum135 CCD camera. All the statistics are compiled in table SX. The structure was solved by the AB-initio method implemented in SHELXD and refined with SHELXL (Sheldrick, G.M. Acta Cryst. A64, 2008, 112-122). Full-matrix least-squares refinement was performed on F2 for all unique reflections, minimizing $\mathrm{w}(\mathrm{Fo} 2-\mathrm{Fc} 2) 2$, with anisotropic displacement parameters for nonhydrogen atoms. The positions of the $\mathrm{H}$ atoms were deduced from coordinates of the non- $\mathrm{H}$ atoms. The non-H atoms were refines with anisotropic temperature parameters.

\section{RESULTS AND DISCUSSION}

\section{Purification protocol}

We recently reported the synthesis of DGDC-based PHUs through reactive extrusion. ${ }^{6,7}$ In this work, DGDC monomer was synthesized in one step from diglycerol and recovered as a white powder, characterized by a melting temperature of $62-69^{\circ} \mathrm{C}$ (Figure 1, pathway 1 ). The application of the recrystallization procedure, recently described by Schimpf et al., to DGDC reaction crude led to the following observation: two different crystalline structures were clearly visible in the flask after $48 \mathrm{~h}$ at $-20{ }^{\circ} \mathrm{C}$ and readily separable (Figure 1, pathway 2 ). The first one is appearing as square like crystals (DGDC-SqC) and the second one as needle like crystals (DGDC-NeC). 


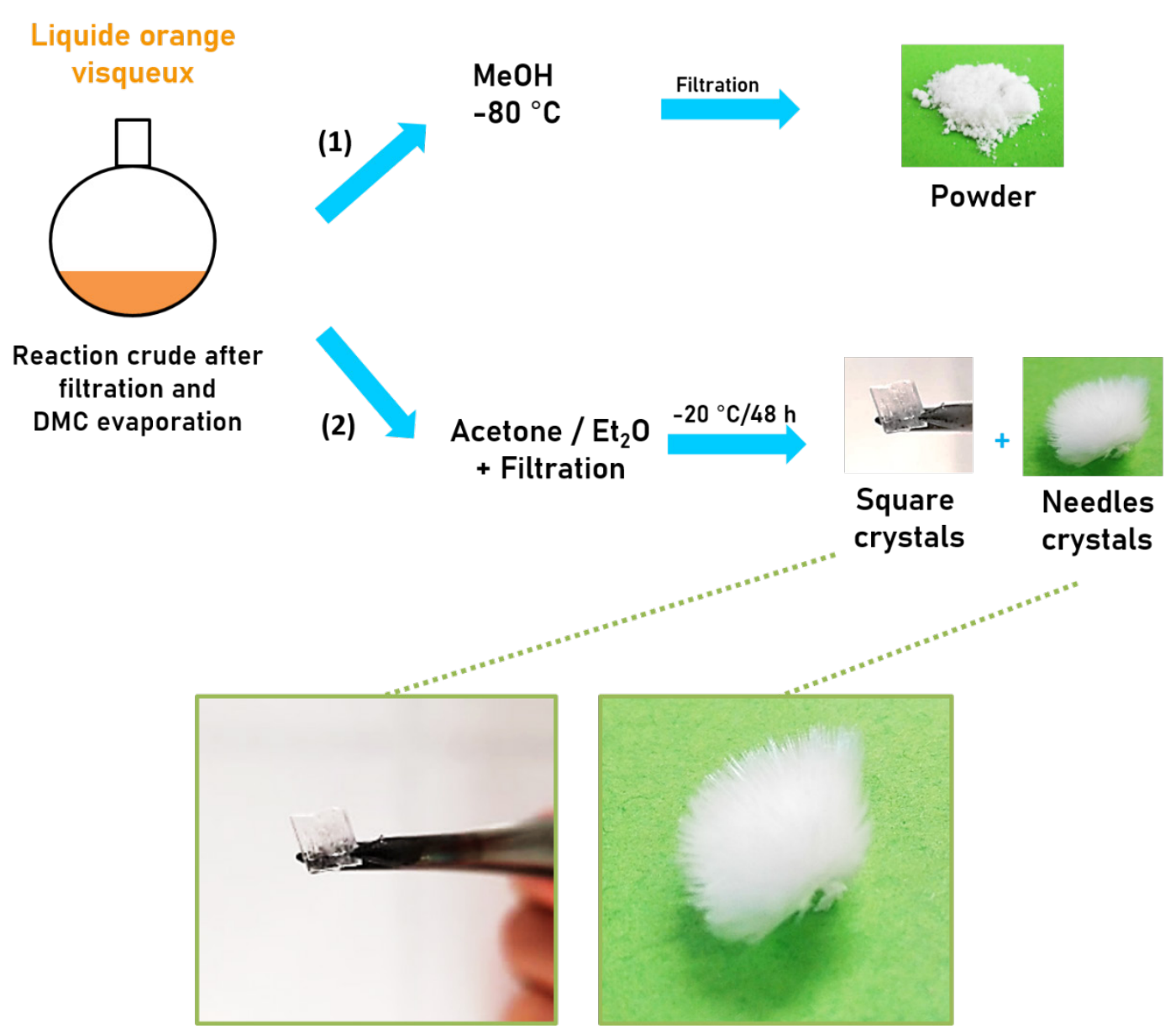

Figure 2. Purification protocols on DGDC reaction crude, after filtration and DMC evaporation. (1) Pathway previously described by Bossion et al ${ }^{5}$ and Magliozzi et al. ${ }^{6}$ (2) Protocol proposed by Schimpf et al. ${ }^{8}$

\section{Characterization of the two species.}

DGDC crystals were first characterized by ESI spectrometry (SI Figure SI.1) The theoretical mass in sodium matrix is $241.03187 \mathrm{~g} / \mathrm{mol}$ and the determined mass were $241.03143 \mathrm{~g} / \mathrm{mol}$ and $241.03149 \mathrm{~g} / \mathrm{mol}$ for DGDC-NeC and DGDC-SqC, respectively, confirming the exact structure of DGDC in each case.

Besides, DGDC-SqC and DGDC-NeC were analyzed by ${ }^{1} \mathrm{H}$ NMR spectroscopy and compared to DGDC-Pow (Figure 2). At first, NMR spectra look similar, but a deeper analysis shows few differences. The signal between 4.2 and $4.3 \mathrm{ppm}$ (green frame, Figure 2) is composed of a double doublet in the case of DGDC-NeC and DGDC-SqC, whereas it appears as a double doublet of doublets in the powder. The latter signal seems to result from an overlay of the 
signals displayed by the two types of crystals. The same phenomenon can be pointed out from the signal corresponding to the $\mathrm{CH}_{2}$ in alpha position of the ether moiety (blue frame, Figure 2).
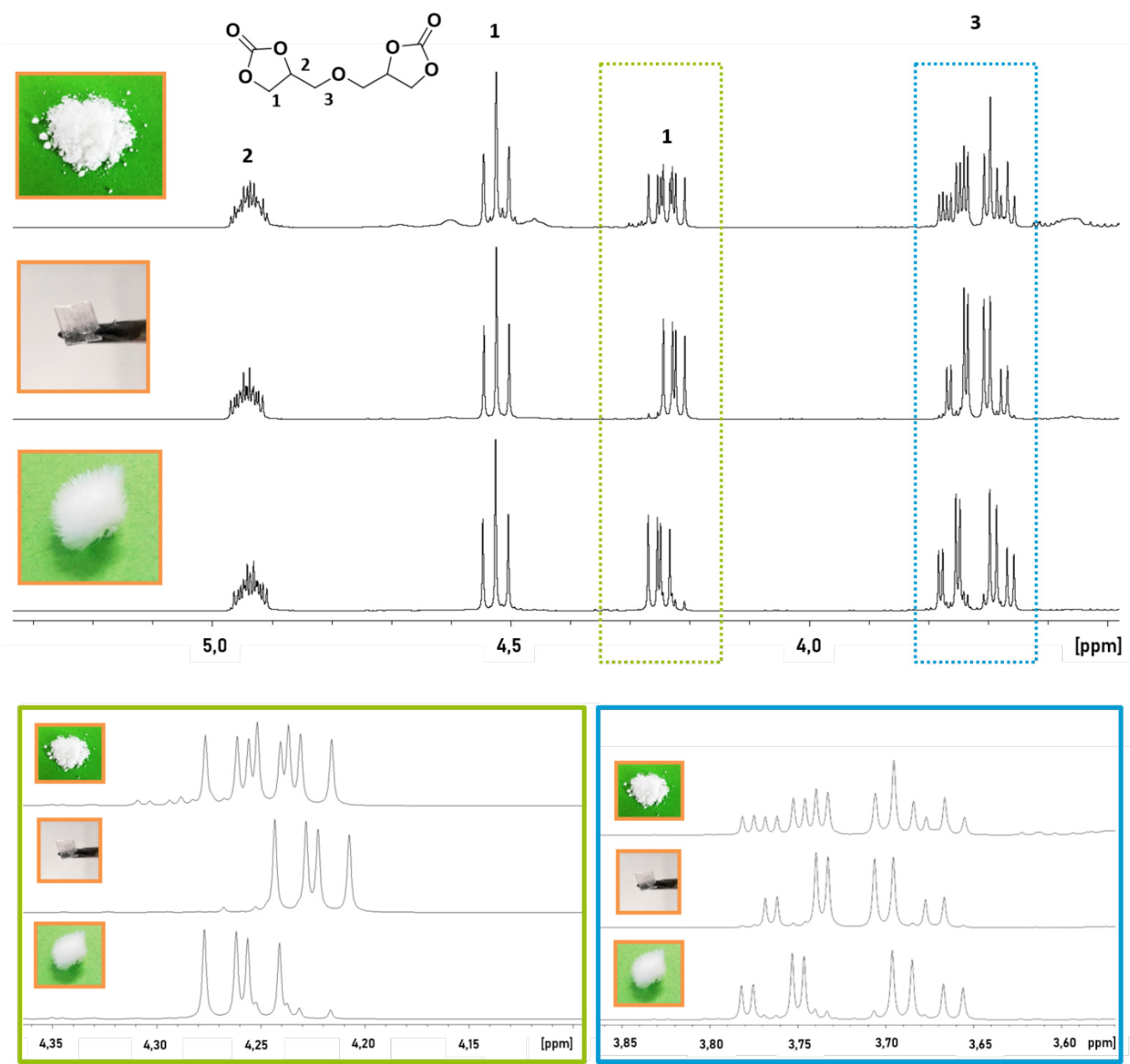

Figure 3. ${ }^{1} \mathrm{H}$ NMR spectra of DGDC powder (top), DGDC square crystals (middle) and DGDC needle crystals (bottom). Main graph: zoom between 3.5 ppm and 5.3 ppm. Zoom-in graphs: zoom between 4.1 ppm and 4.36 ppm (left) and between $3.57 \mathrm{ppm}$ and $3.86 \mathrm{ppm}$ (right).

Figure 3 shows ${ }^{13} \mathrm{C}$ NMR spectrum of DGDC species, with specific zoom-in zones, comparing the signals obtained for DGDC-SqC, DGDC-NeC and DGDC-pow, respectively. As previously mentioned, the spectrum corresponding to the powder contains double signals, resulting from 
an overlay of the signals corresponding to the square and DGDC-NeC. In the two crystal cases, the signals appear single and slightly shifted on respective spectra.

The last results obtained from NMR analysis led to the first conclusion that DGDC-Pow must be a mixture of DGDC-SqC and DGDC-NeC, with a ratio close to 50/50.
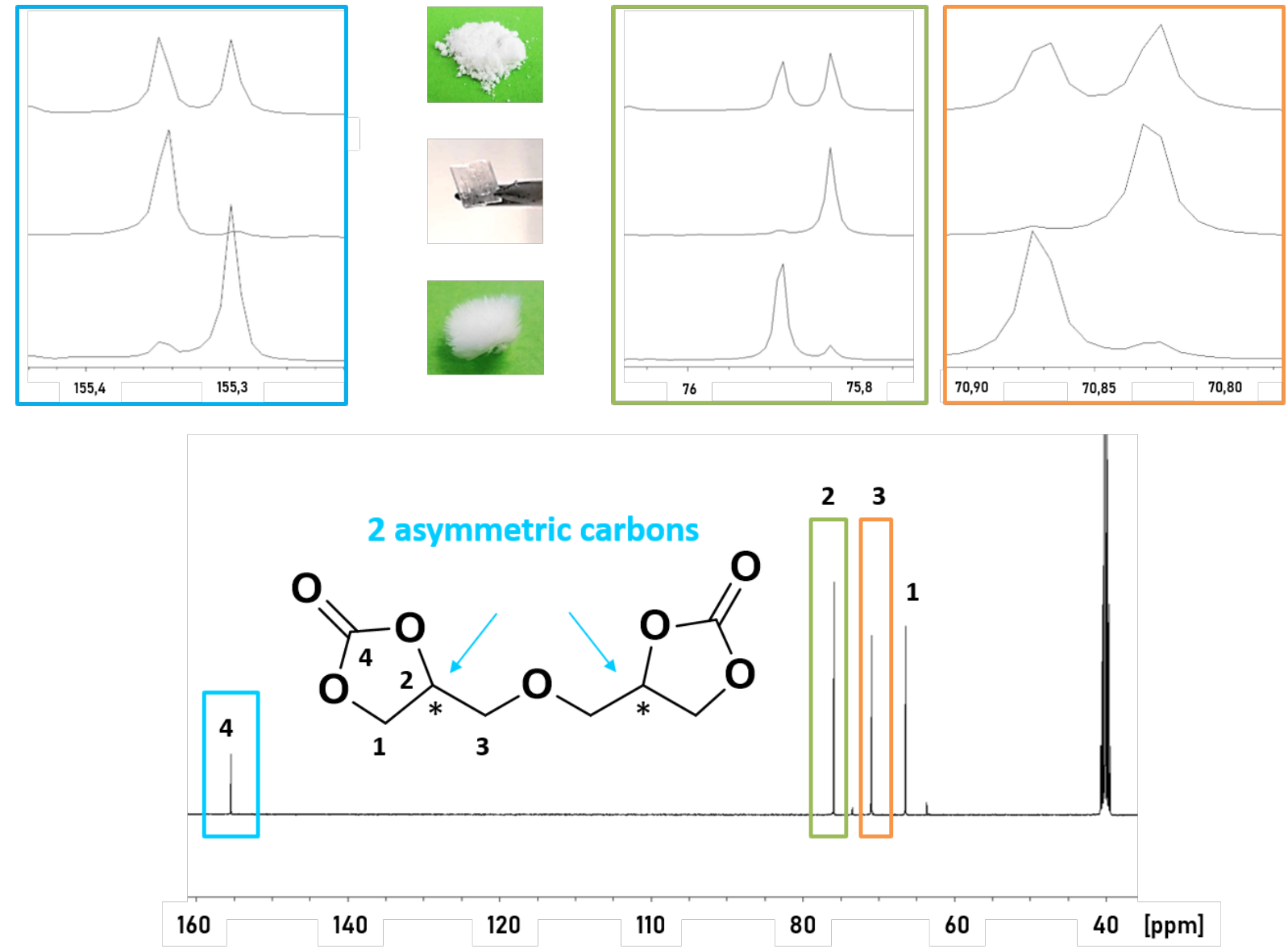

Figure 4. ${ }^{13}$ C NMR spectrum of DGDC (bottom) and zoom-in graphs of stacked spectra of power (top), square crystals (middle) and DGDC-NeC (bottom), between 155.22 and $115.44 \mathrm{ppm}$ (left), between 75.775 and $76.25 \mathrm{ppm}$ (middle) and 70.78 and $70.90 \mathrm{ppm}$ (right).

XRD analyses were performed on the two different crystals; results regarding crystal system parameters are summarized in Figure 4. DGDC-SqC are characterized by an orthorhombic crystalline mesh and were identified as R,R and S,S enantiomers. In this structure, the carbonate rings are oriented in opposite directions, regarding to the ether moiety. On the contrary, DGDC$\mathrm{NeC}$ are crystallizing according to a monoclinic crystalline mesh and were found to correspond to $\mathrm{R}, \mathrm{S}$ and $\mathrm{S}, \mathrm{R}$ enantiomers. In the last structure, the rings are oriented in the same direction 
regarding the ether bond. A table gathering the different crystal system parameters is available in SI, Table SI.2.
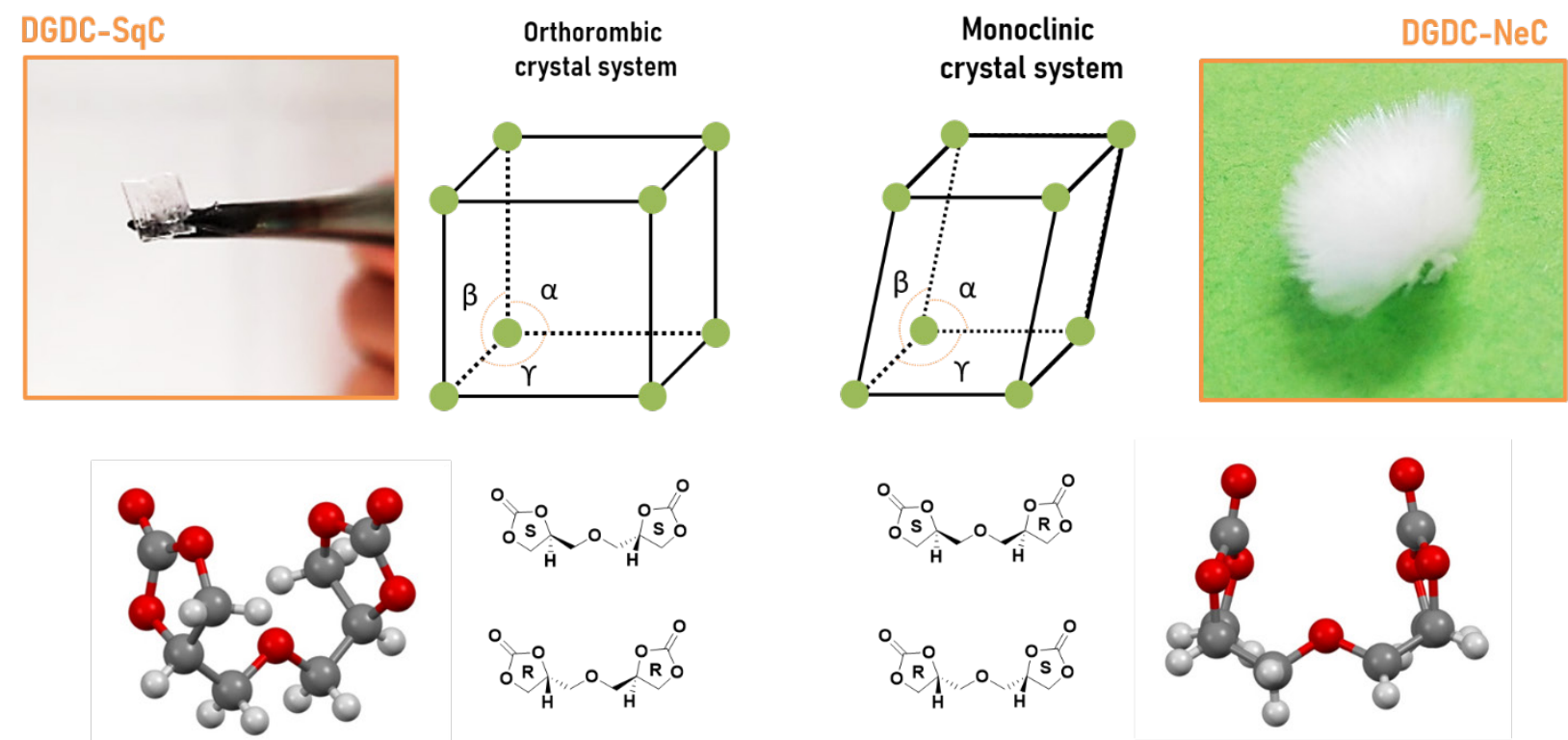

$R, R$ et $S, S$ Enantiomers

R,S et S,R Enantiomers

Figure 5. XRD analysis of DGDC-NeC and DGDC-SqC: pictures of the species, $3 D$ images of identified structures and Cram schemes of the different isomers.

In order to further investigate the small shift differences calculated on ${ }^{1} \mathrm{H}$ NMR signals of DGDC-SqC and DGDC-NeC and properly attribute the triplet signal at $4.52 \mathrm{ppm}$, the double doublet between 4.2 and $4.3 \mathrm{ppm}$ and the double doublet of doublets between 3.65 and $3.8 \mathrm{ppm}$, coupling constants were measured on ${ }^{1} \mathrm{H}$ NMR spectra and Karplus method was applied (Table SI.3 and Figures SI.2.a-d). Angles were calculated between protons labelled $\mathrm{H}_{1}$ or $\mathrm{H}_{3}$ and proton $\mathrm{H}_{2}$ (Figure 2 and Figure 5), using measured coupling constants from DGDC ${ }^{1} \mathrm{H}$ NMR signals. Protons in $\mathrm{H}_{1}$ and $\mathrm{H}_{3}$ groups were labelled $\mathrm{H}_{1 \text { or }}{ }^{\text {cis }}$ and $\mathrm{H}_{1 \text { or } 3}{ }^{\text {trans }}$ respectively for the proton in upward or backward position relatively to the carbonate cycle (Scheme on top of Figure 5). Thanks to this ${ }^{1} \mathrm{H}$ NMR study and considering dihedral angles (conformers in solution), triplet signal at $4.52 \mathrm{ppm}$ was attributed to $\mathrm{H}_{1}{ }^{\text {cis }}$, for both DGDC-SqC and DGDC-NeC structures, the double doublet at 4.23 or 4.26 (DGDC-SqC and DGDC-NeC respectively) was found to be related to $\mathrm{H}_{1}{ }^{\text {trans }}$. Besides, the multiplet between 3.65 and $3.80 \mathrm{ppm}$ in DGDC-Pow, was found 
to be resulting from overlay of the double doublet of doublet signals of the different stereoisomers. Indeed, the signals at 3.66 and $3.69 \mathrm{ppm}(\mathrm{DGDC}-\mathrm{SqC})$ and 3.67 and $3.70 \mathrm{ppm}$ (DGDC-NeC) are related to the proton $\mathrm{H}_{3}{ }^{\mathrm{cis}}$ whereas the signals at 3.75 and 3.78 ppm (DGDC$\mathrm{SqC}$ ) and 3.74 and $3.77 \mathrm{ppm}$ (DGDC-NeC) are corresponding to proton $\mathrm{H}_{3}{ }^{\text {trans }}$. Finally, for the first time, the entire ${ }^{1} \mathrm{H}$ NMR spectrum of DGDC could be assigned.

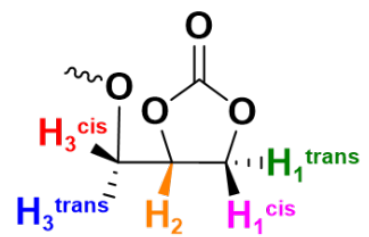

\section{DGDC-NeC}

\section{$\mathrm{H}_{2}$}
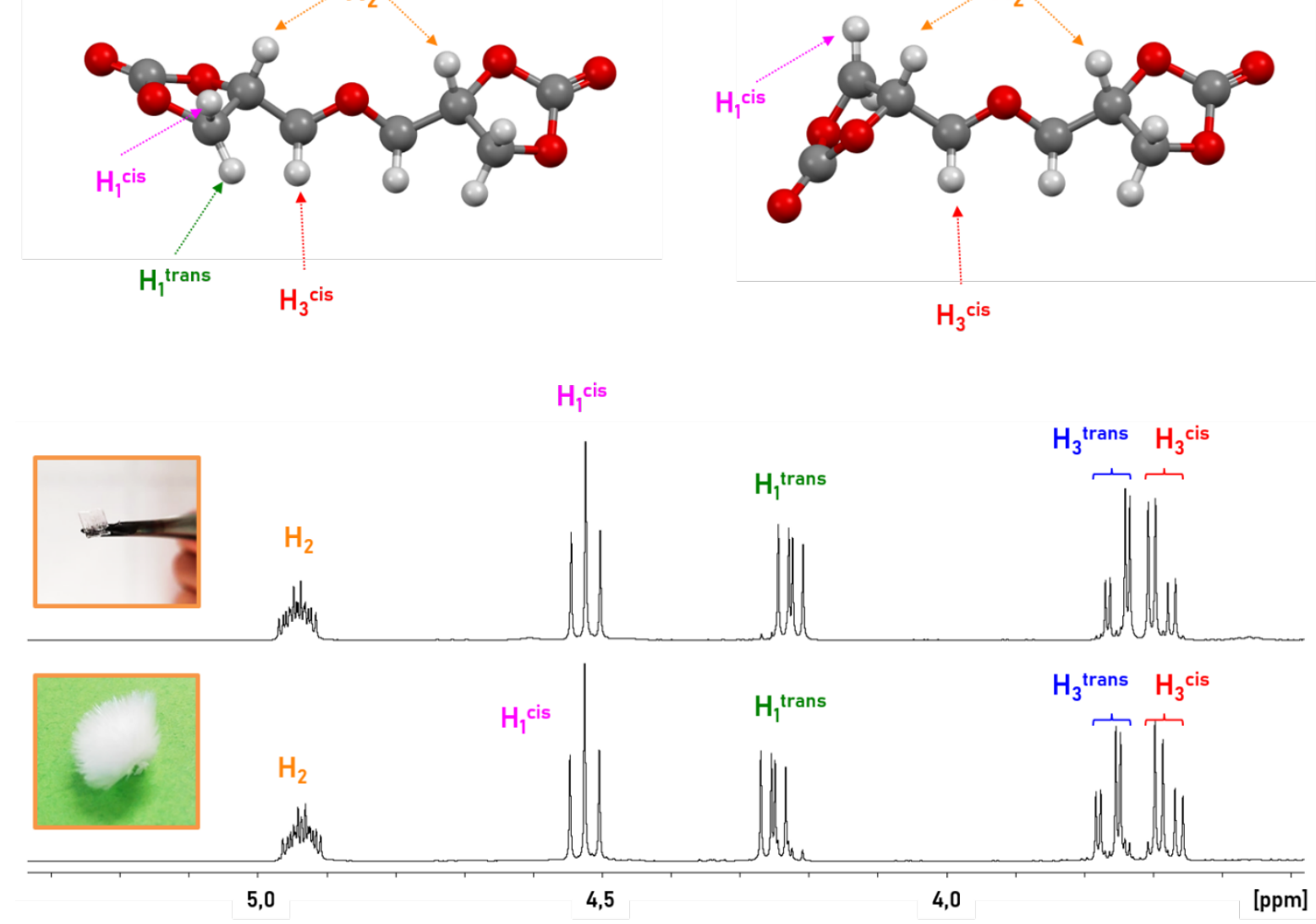

\section{DGDC-SqC}

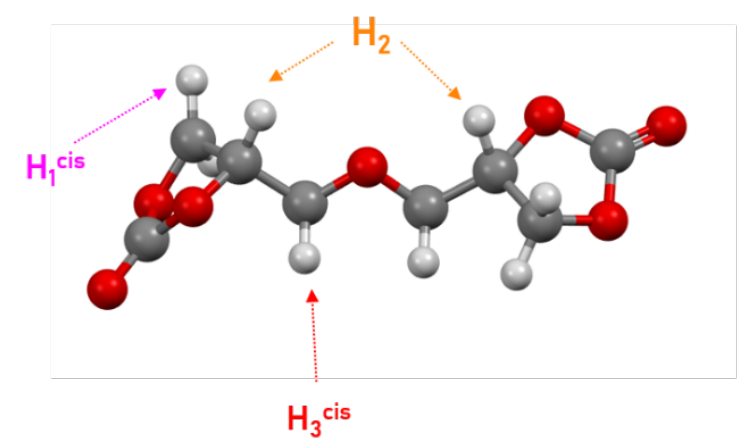

Figure 6. 3D representations of DGDC-NeC and DGDC-SqC with angles calculated from Karplus methods, related to solution conformation. Final attribution of all DGDC protons, labelled $\mathrm{H}_{1}{ }^{\text {cis }}, \mathrm{H}_{\mathrm{I}}{ }^{\text {trans }}, \mathrm{H}_{2}, \mathrm{H}_{3}{ }^{\text {cis }}, \mathrm{H}_{3}{ }^{\text {trans }}$, as represented on bottom left scheme.

Additionally, the two DGDC-SqC and DGDC-NeC were analyzed by DSC and the thermograms, compared with DGDC-pow, (Figure 5). DGDC-NeC and DGDC-SqC are 
characterized by melting temperatures of 70.2 and $105{ }^{\circ} \mathrm{C}$ respectively, whereas the melting temperature of DGDC-pow was identified around $62-63{ }^{\circ} \mathrm{C}$. The high melting temperature obtained for DGDC-SqC is the highest value ever reported in literature.

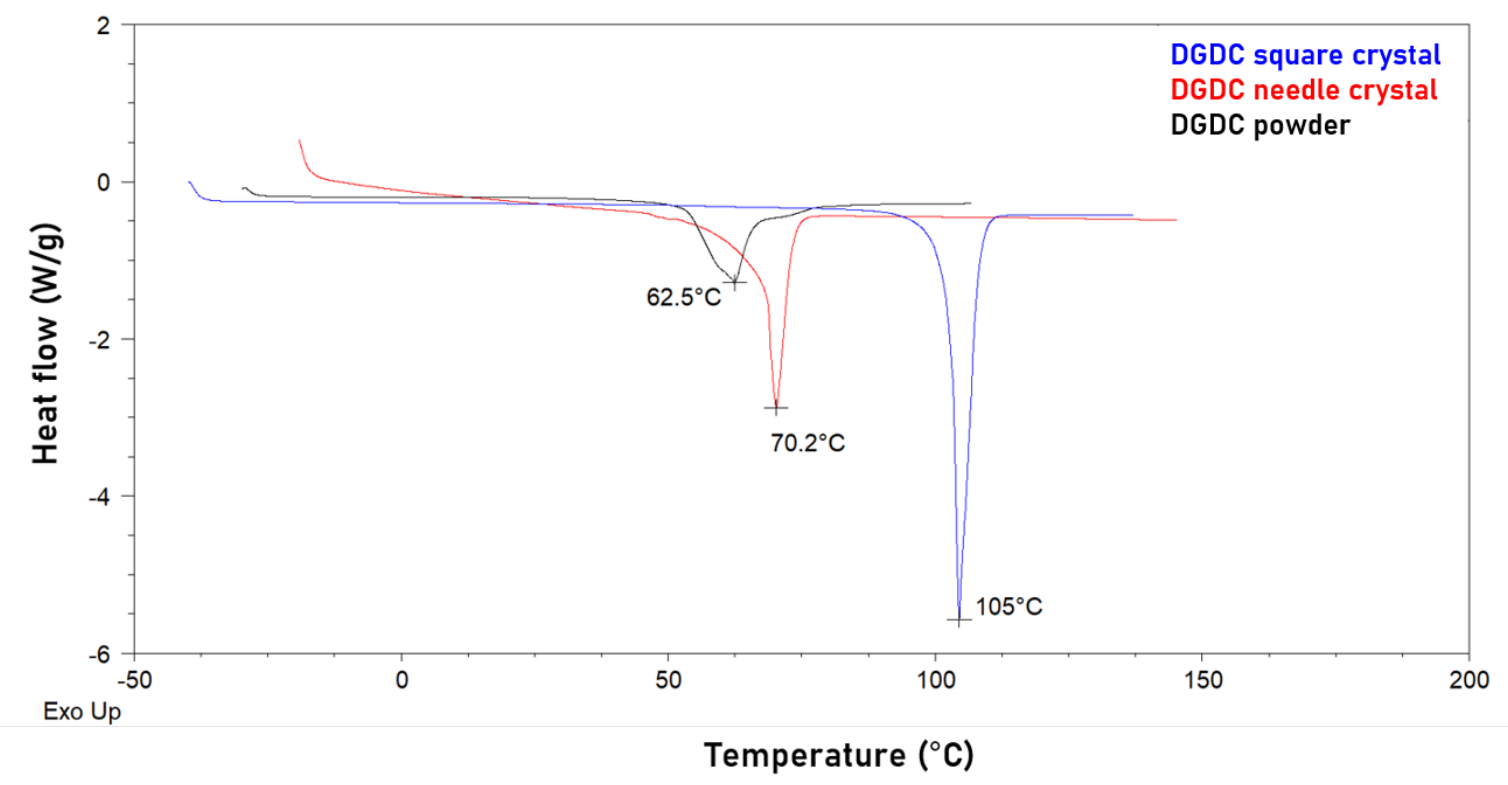

Figure 7.First heating cycle obtained in DSC analysis of DGDC-NeC (red curve), DGDC-SqC (blue) and DGDC-pow (black).

DGDC-Pow, previously assumed as a mixture of DGDC-SqC and DGDC-NeC is characterized by the lowest melting temperature among the three species. In order to explain this result, the melting behavior of DGDC-SqC/DGDC-NeC mixtures was investigated. DSC first heating cycles were recorded on mixtures composed of different amounts of DGDC-SqC and DGDC$\mathrm{NeC}$. As clearly visible on Figure 6 (left), the higher the amount of DGDC-SqC in the mixture, the higher the melting temperature. Besides, the melting temperatures of the mixture with intermediate compositions seems to proceed in different steps (up to 3 melting points identified on the DSC first heating cycle), possibly suggesting different crystal organizations. As previously identified, DGDC-Pow is characterized (Figure 6, left) by a melting temperature around $63{ }^{\circ} \mathrm{C}$, confirming that the ratio DGDC-SqC/DGDC-NeC in DGDC-Pow is close to 50/50. According to the different melting points determined thanks to this DSC study, the phase 
diagram of the mixture was drawn, assuming an azeotropic behaviour (Figure 6, right). These results led to the following conclusion: DGDC-Pow is composed of a DGDC-SqC/DGDC-NeC mixture with a ratio around 50/50 and its lower melting temperature is explained by the azeotropic behavior of the blend.
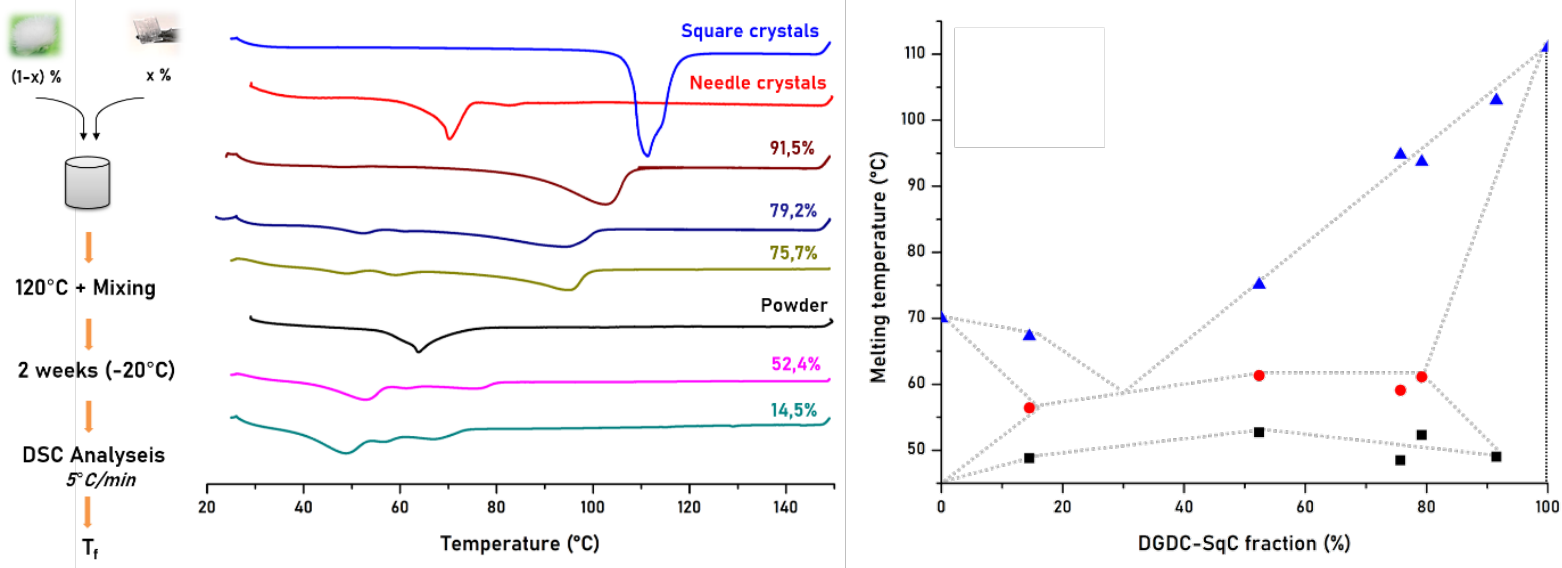

Figure 8. Left: DGDC-SqC and DGDC-NeC blending procedure, prior to DSC analysis. DSC first heating cycle curves obtained for systems containing different DGDC-SqC/DGDC-NeC ratios. The percentage values indicate the mass proportion of DGDC-SqC in the mixture. Right: Possible phase diagram of the mixture DGDC-SqC/DGDC-NeC, drawn according to melting point identified in DSC study.

Additionally, thermal stabilities and temperature behavior of the three DGDC species were investigated by TGA analysis. Not surprisingly, DGDC-Pow demonstrated the lowest thermal stability $\left(\mathrm{T}_{\mathrm{d}}{ }^{5 \%}=173{ }^{\circ} \mathrm{C}\right)$ compared to DGDC-NeC $\left(\mathrm{T}_{\mathrm{d}}{ }^{5 \%}=197{ }^{\circ} \mathrm{C}\right)$ and DGDC-SqC $\left(\mathrm{T}_{\mathrm{d}}{ }^{5 \%}=\right.$ $204^{\circ} \mathrm{C}$ ). TGA weight curves and detailed thermal study are available in SI.3 (Figure SI.3.a-b and Table SI.4-5).

\section{Separation protocol.}

The recrystallization in acetone/ $\mathrm{Et}_{2} \mathrm{O}$ allows obtaining mixture of DGDC-NeC and DGDC-SqC crystals in the flask. Illustration of this phenomenon is available in SI, Figure SI-4.a. In order to overcome this issue, a two-step protocol was developed. First, the formation of DGDC-SqC was directly induced from reaction crude (after solvent evaporation) at $18^{\circ} \mathrm{C}$, leading to crystals recovery after 3 days. Secondly, a mixture of acetone and $\mathrm{Et}_{2} \mathrm{O}$ was added to the reaction crude 
residue (after removal of DGDC-SqC) and DGDC-NeC could be recovered after 2 days at $20^{\circ} \mathrm{C}$, as illustrated in Figure 9 (details in materials and methods section). Another recrystallization method, based on the same two solvents and describing the specific DGDCSqC recovery is also detailed in SI (Figure SI.5.b).

Thanks to this procedure, DGDC-SqC and DGDC-NeC were easily separated. It is worth noting that DGDC-SqC could be recovered directly from the reaction crude, without requiring any solvent addition.

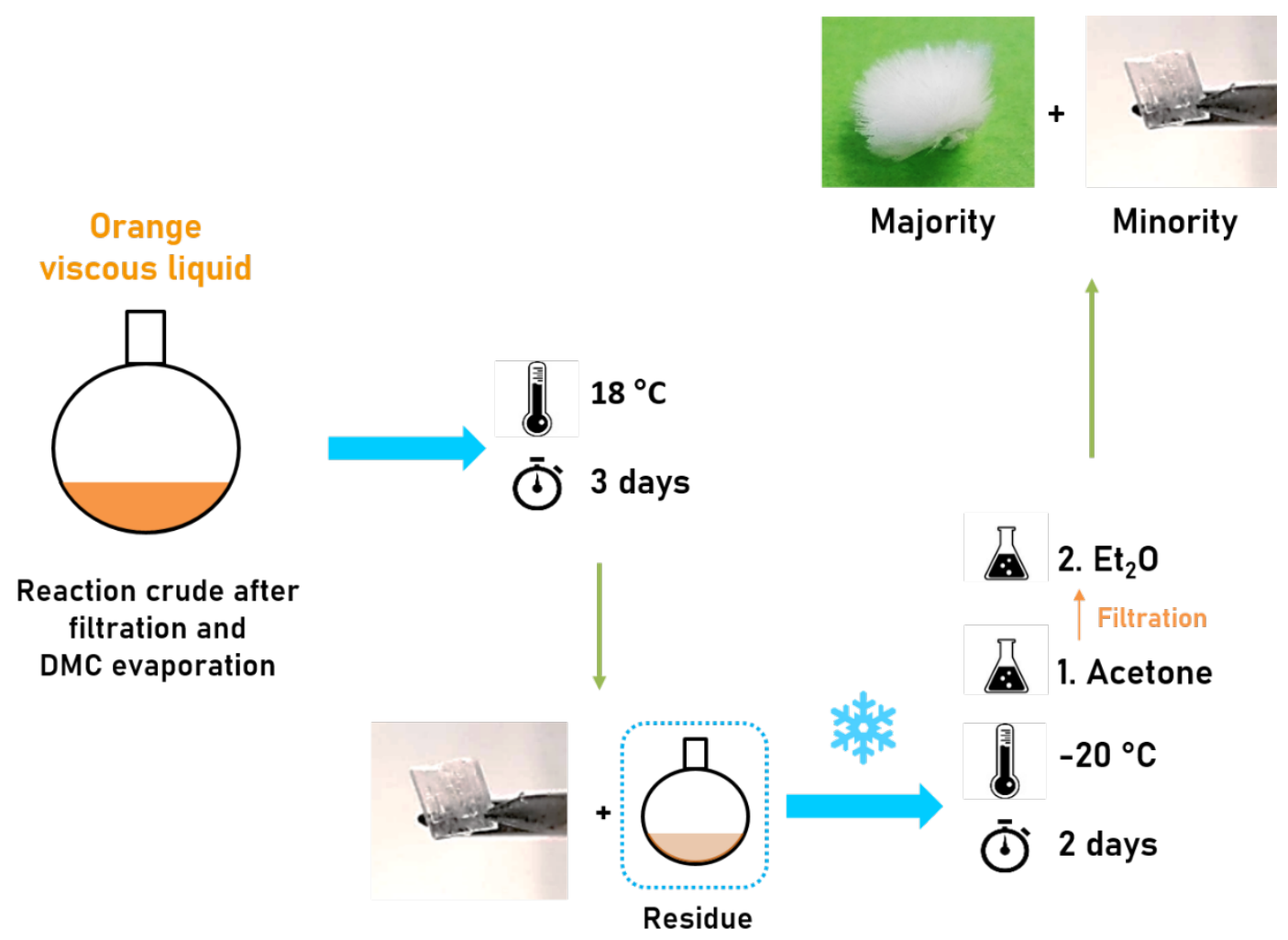

Figure 9. Recrystallization protocol allowing the separated obtaining of DGDC-SqC and the DGDC-NeC.

\section{Application to PHU synthesis.}

Once the two crystalline species recovered in sufficient amounts, the latter were first reacted with hexylamine and afterwards in the course of PHUs bulk synthesis. Model reactions between the different DGDC species and hexylamine were followed by in-situ ${ }^{1} \mathrm{H}$ NMR in DMSO-d6. The reactions were performed at $50{ }^{\circ} \mathrm{C}$, over $20 \mathrm{~h}$ and the conversion was calculated according 
to the disappearance of signals corresponding to carbonate moieties. The curves representing the conversion over time and associated kinetic constants are displayed in Figure 10. Conversions of DGDC-SqC and DGDC-NeC as well as calculated reaction kinetic constants, 1.71 and $1.63 \mathrm{~L} \cdot \mathrm{mol}^{-1} \cdot \mathrm{h}^{-1}$, respectively suggests very similar reactivity in solution of the cyclic carbonate moieties, irrespective of the crystal species.

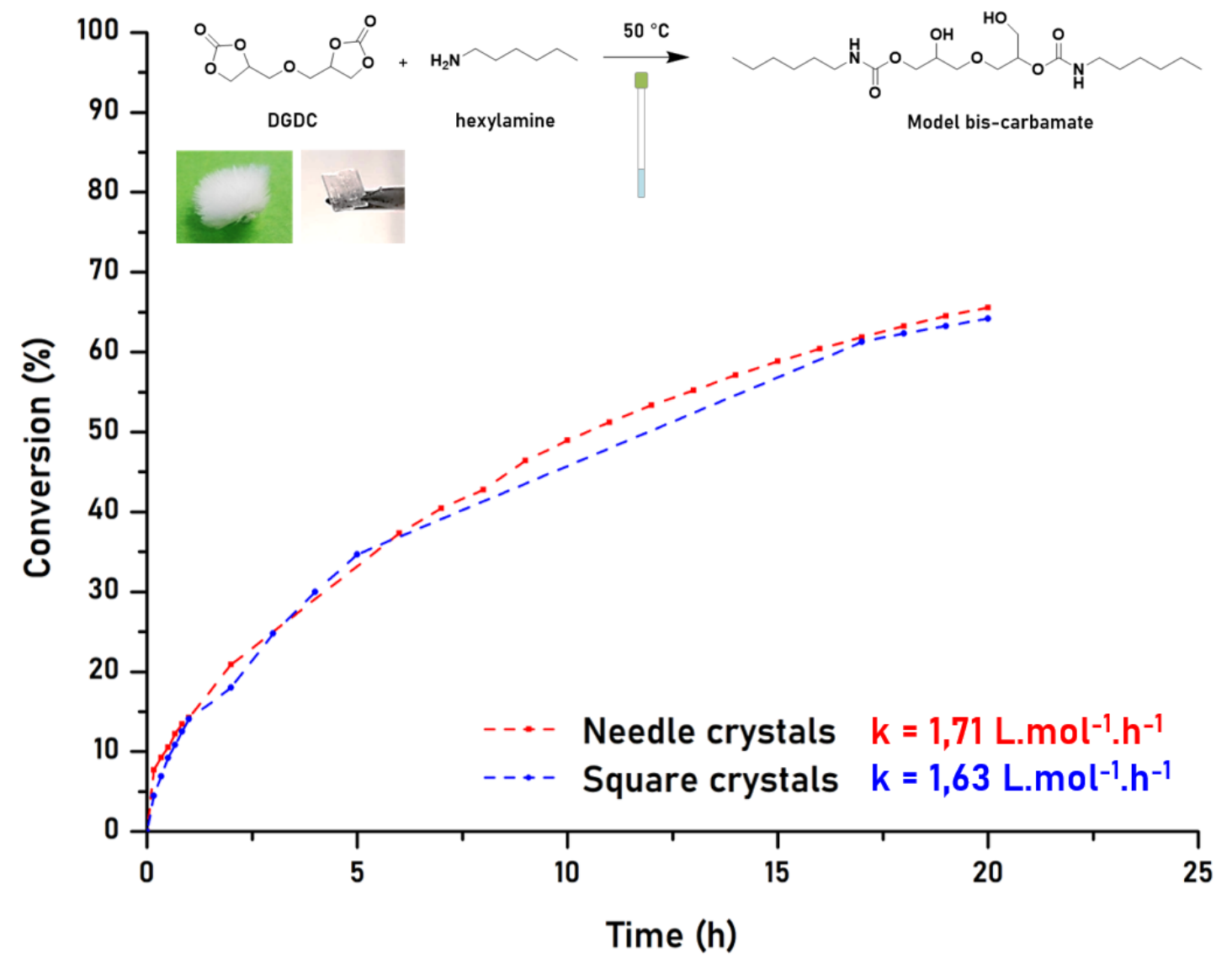

Figure 10. Model kinetic of the reaction between DGDC-SqC and DGDC-NeC with hexylamine, followed by ${ }^{l} H N M R\left(50{ }^{\circ} \mathrm{C}\right.$, 1 mol.L-1 in DMSO-d6, ratio 1:1). Blue and red curves respectively correspond to model reaction performed with DGDC$\mathrm{SqC}$ and $\mathrm{DGDC}-\mathrm{NeC}$ species.

Finally, DGDC-SqC, DGDC-NeC and DGDC-Pow were tested during bulk PHU synthesis, involving 6DA, 10DA and m-xylylene diamine, leading to 9 polymers, named PHUs 1-9. The PHUs were synthesized through mechanical stirring and a temperature of $110^{\circ} \mathrm{C}$ was set in order to ensure the correct melting of DGDC-SqC. The final PHUs were analyzed by NMR, 
SEC, DSC, TGA (Table 1) and MALDI (Figure SI.6.a-b). Figure 11 displays the reaction conditions and compared chromatograms of the PHUs obtained for each DGDC species and diamine involved. As clearly evidenced, the highest molar masses were obtained with DGDCSqC-based PHUs (blue curves) and the lowest for DGDC-NeC-based PHUs (black curves). Besides, DGDC-Pow-based PHU provided intermediate results. This last observation shows that, in bulk polymerization, the stereochemistry of DGDC-SqC, meaning the DGDC structure in which the two carbonate cycles are oriented in opposite directions regarding the ether moiety, favors the diamine and chain insertion leading to higher polymerization degree. Besides, NMR spectra (Example of PHUs4-6, Figure SI.5) allowed carbonate conversion calculation and $\mathrm{OH}(\mathrm{I}) / \mathrm{OH}(\mathrm{II})$ determination. $\mathrm{OH}(\mathrm{I})$ fraction is generally around $30 \%$. Nevertheless, in the cases of polymerizations with 10DA and 6DA, $\mathrm{OH}(\mathrm{I})$ fraction is more important (up to $36.5 \%$ ) when DGDC-NeC is used as precursor. On the contrary, in the cases of polymerizations with xy-DA, the $\mathrm{OH}(\mathrm{I})$ fractions remain comparable, whatever the DGDC precursor. In addition, thermal characterization of $\mathrm{PHU}_{1-9}$ demonstrated comparable thermal stabilities (close values of $\left.\mathrm{T}_{\mathrm{d} 5 \%}\right)$ and variable $\mathrm{T}_{\mathrm{g}}$. Indeed, $\mathrm{T}_{\mathrm{g}}$ increase as molar masses increase, with highest values, in each case, when DGDC-SqC was employed.

Besides, despite the fact that polymerizations were conducted at $110{ }^{\circ} \mathrm{C}$, the amount of urea generated remains relatively low, excepted in the case of $\mathrm{PHU}_{6}(10 \mathrm{DA})$.

Finally, these results demonstrate the importance of DGDC stereochemistry in the course of PHUs bulk syntheses. Indeed, the opposite rings configuration observed in the case of DGDC$\mathrm{SqC}$ seems to favor the nucleophilic attack of the amine and the chain insertion, for reaction occurring in the melt phase. In addition, this phenomenon, is eventually exacerbated depending on the diamine structure. This study, performed on three different diamines shows that the shorter the diamine chain length (with the lower steric hindrance), the higher the effect of DGDC stereochemistry. Indeed, the molar mass difference between PHUs synthesized from 
DGDC-Sqc and DGDC-NeC in the case of 6DA (lowest steric hindrance) is the highest one ( $10800 \mathrm{~g} / \mathrm{mol})$ whereas it is the lowest one in the case of the most sterically hindered diamine, $\mathrm{xy}-\mathrm{DA}(450 \mathrm{~g} / \mathrm{mol})$.
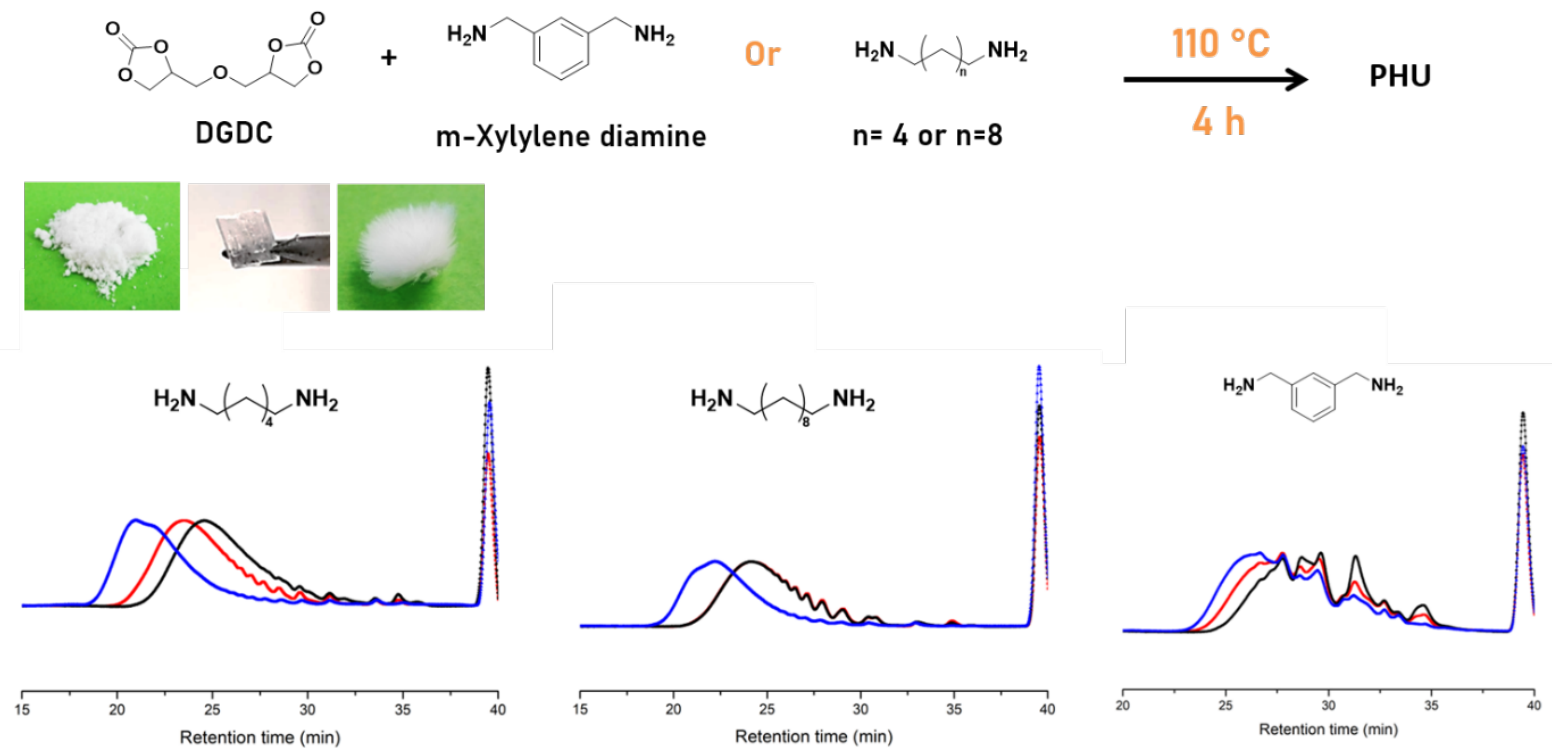

Figure 11. Application of DGDC-SqC and DGDC-NeC in the course of PHU bulk synthesis with mechanical stirring. Comparison of the SEC traces (DMF, toluene as flow marker) obtained for each DGDC species, relatively to the diamine involved.

Table 1. DGDC-based PHUs synthesized from DGDC-NeC, DGDC-SqC or DGDC-Pow and associated properties.

\begin{tabular}{|c|c|c|c|c|c|c|c|c|}
\hline Diamine & PHU & DGDC & $\begin{array}{c}\text { Conversion } \\
(\%)^{\mathrm{a}}\end{array}$ & Urethane/Urea ${ }^{a}$ & $\begin{array}{c}\mathrm{OH}(\mathrm{I}) / \mathrm{OH} \\
\text { (II) }\end{array}$ & $\mathbf{M}_{\mathbf{n}}[\boldsymbol{Ð}]^{\mathbf{b}}$ & $\begin{array}{c}\mathbf{T}_{\mathbf{g}} \\
\left({ }^{\circ} \mathbf{C}\right) \\
\mathbf{c}\end{array}$ & $\begin{array}{l}\mathbf{T}_{\mathrm{d} 5} \% \\
\left({ }^{\circ} \mathrm{C}\right)^{\mathrm{d}}\end{array}$ \\
\hline \multirow{3}{*}{ 6DA } & 1 & Powder & 98.55 & $99.05 / 0.95$ & $28 / 72$ & $5000[2.5]$ & 20.1 & 213 \\
\hline & 2 & $\begin{array}{l}\text { Needles } \\
\text { Crystals }\end{array}$ & 99.15 & $99.47 / 0.53$ & $36.5 / 63.5$ & $3200[2.4]$ & 17.3 & 222 \\
\hline & 3 & Square Crystals & 98.11 & $98.15 / 1.85$ & $29.3 / 70.6$ & 14000 [2.6] & 21.5 & 220 \\
\hline \multirow{3}{*}{ 10DA } & 4 & Powder & 99.66 & $99.27 / 0.73$ & $26.6 / 73.8$ & $4260[2.2]$ & 10.0 & 213 \\
\hline & 5 & $\begin{array}{l}\text { Needles } \\
\text { Crystals }\end{array}$ & 99.84 & $100 / 0$ & $33 / 66$ & $4100[2.2]$ & 8.3 & 215 \\
\hline & 6 & Square Crystals & 99.42 & $90.17 / 9.83$ & $25.3 / 74.7$ & $11500[2.2]$ & 14.3 & 220 \\
\hline \multirow{3}{*}{ Xy-DA } & 7 & Powder & 84.21 & $92.04 / 7.96$ & $31.9 / 68.1$ & $1200[2.0]$ & 29.7 & 216 \\
\hline & 8 & $\begin{array}{l}\text { Needles } \\
\text { Crystals }\end{array}$ & 78.10 & $96.25 / 3.75$ & $30.3 / 69.7$ & $950 \mid 1.9]$ & 24.7 & 211 \\
\hline & 9 & Square Crystals & 89.89 & $96.59 / 3.41$ & $30.7 / 69.3$ & $1400[2.2]$ & 36.0 & 210 \\
\hline
\end{tabular}

${ }^{\mathrm{a}}$ Determined by ${ }^{1} \mathrm{H}$ NMR in DMSO-d6 at room temperature. ${ }^{\mathrm{b}}$ Determined by SEC in DMF, with LibR salts, toluene as flow marker and calculated relatively to PS standards. ${ }^{\mathrm{c}}$ Determined from DSC second heating cycle, $-40{ }^{\circ} \mathrm{C}$ to $180{ }^{\circ} \mathrm{C}$ at $10{ }^{\circ} \mathrm{C} / \mathrm{min}$. ${ }^{\mathrm{d}}$ Determined by TGA analysis, from room temperature to $600{ }^{\circ} \mathrm{C}$ under $\mathrm{N}_{2}$ atmosphere at $10{ }^{\circ} \mathrm{C} / \mathrm{min}$. 


\section{CONCLUSION}

This study demonstrated that DGDC stereoisomers crystallize according to different crystal systems, appearing as square transparent crystals and needle like structures. Besides, it has been proved that the white powder classically obtained for DGDC was actually resulting from the mixing of stereoisomers, and consequently the blending of the two crystal systems. The two DGDC shapes not only show differences in terms of signals on NMR spectra, but are also characterized by very specific physicochemical properties, such as melting temperature. Finally, our work pointed out the impact of this stereochemistry on polyhydroxyurethane properties, shining a light on the benefit brought by DGDC-SqC on PHUs molar masses and $\mathrm{T}_{\mathrm{gS}}$. 


\section{BIBLIOGRAPHY}

(1) Schimpf, V.; Max, J. B.; Stolz, B.; Heck, B.; Mülhaupt, R. Semicrystalline Non-Isocyanate Polyhydroxyurethanes as Thermoplastics and Thermoplastic Elastomers and Their Use in 3D Printing by Fused Filament Fabrication. Macromolecules 2019, 52 (1), 320-331.

(2) Rokicki, G.; Kuran, W. Cyclic Carbonates Obtained by Reactions of Alkali Metal Carbonates with Epihalohydrins. Bull. Chem. Soc. Jpn. 1984, 57 (6), 1662-1666.

(3) Tryznowski, M.; widerska, A.; Zołek-Tryznowska, Z.; Gołofit, T.; Parzuchowski, P. G. Facile Route to Multigram Synthesis of Environmentally Friendly Non-Isocyanate Polyurethanes. Polymer (Guildf). 2015, 80, 228-236.

(4) Van Velthoven, J. L. J.; Gootjes, L.; Van Es, D. S.; Noordover, B. A. J.; Meuldijk, J. Poly(Hydroxy Urethane)s Based on Renewable Diglycerol Dicarbonate. Eur. Polym. J. 2015, 70, 125-135.

(5) Bossion, A.; Aguirresarobe, R. H.; Irusta, L.; Taton, D.; Cramail, H.; Grau, E.; Mecerreyes, D.; Su, C.; Liu, G.; Müller, A. J.; et al. Unexpected Synthesis of Segmented Poly(Hydroxyurea-Urethane)s from Dicyclic Carbonates and Diamines by Organocatalysis. Macromolecules 2018, 51 (15), 5556-5566.

(6) Magliozzi, F.; Chollet, G.; Grau, E.; Cramail, H. Benefit of the Reactive Extrusion in the Course of Polyhydroxyurethanes Synthesis by Aminolysis of Cyclic Carbonates. ACS Sustain. Chem. Eng. 2019, 7 (20), 17282-17292.

(7) Magliozzi, F.; Scali, A.; Chollet, G.; Montarnal, D.; Grau, E.; Cramail, H. Hydrolysable Bio-Based Polyhydroxyurethane Networks with Shape Memory Behavior at Body Temperature. ACS Sustain. Chem. Eng. 2020, 8 (24), 9125-9135.

(8) Schimpf, V.; Heck, B.; Reiter, G.; Mülhaupt, R. Triple-Shape Memory Materials via Thermoresponsive Behavior of Nanocrystalline Non-Isocyanate Polyhydroxyurethanes. Macromolecules 2017, 50 (9), 3598-3606. 